\title{
Pore-scale simulation of fluid flow and solute dispersion in three-dimensional porous media
}

\author{
Matteo Icardi \\ Division of Computer, Electrical and Mathematical Science and Engineering, King Abdullah University of Science and Technology, \\ 23955-6900, Thuwal, Saudi Arabia and Institute for Computational Engineering and Sciences, \\ University of Texas at Austin, 78712-0027, Austin, Texas, USA \\ Gianluca Boccardo and Daniele L. Marchisio* \\ Department of Applied Science and Technology, Politecnico di Torino, 10129 Torino, Italy \\ Tiziana Tosco and Rajandrea Sethi \\ Department of Environment, Land and Infrastructures, Politecnico di Torino, 10129 Torino, Italy
}

(Received 18 March 2014; published 31 July 2014)

\begin{abstract}
In the present work fluid flow and solute transport through porous media are described by solving the governing equations at the pore scale with finite-volume discretization. Instead of solving the simplified Stokes equation (very often employed in this context) the full Navier-Stokes equation is used here. The realistic three-dimensional porous medium is created in this work by packing together, with standard ballistic physics, irregular and polydisperse objects. Emphasis is placed on numerical issues related to mesh generation and spatial discretization, which play an important role in determining the final accuracy of the finite-volume scheme and are often overlooked. The simulations performed are then analyzed in terms of velocity distributions and dispersion rates in a wider range of operating conditions, when compared with other works carried out by solving the Stokes equation. Results show that dispersion within the analyzed porous medium is adequately described by classical power laws obtained by analytic homogenization. Eventually the validity of Fickian diffusion to treat dispersion in porous media is also assessed.
\end{abstract}

DOI: 10.1103/PhysRevE.90.013032

PACS number(s): 47.56.+r, 47.11.Df, 47.10.ad, 05.60.Cd

\section{INTRODUCTION}

Flow and dispersion processes in porous media are found in many important industrial, biological, and environmental applications and have been studied intensively over many decades. At the microscopic scale (or pore scale) these phenomena are governed by the fundamental continuity equation, that for an incompressible fluid reads as follows [1]:

$$
\nabla \cdot \mathbf{v}=0
$$

the Navier-Stokes equation:

$$
\frac{\partial \mathbf{v}}{\partial t}+\mathbf{v} \cdot \nabla \mathbf{v}=-\frac{\nabla p}{\rho}+\mathbf{g}+v \nabla \cdot\left(\nabla \mathbf{v}+\nabla \mathbf{v}^{\mathrm{T}}\right)
$$

and the advection-diffusion equation:

$$
\frac{\partial c}{\partial t}+\mathbf{v} \cdot \nabla c=\nabla \cdot\left(D_{m} \nabla c\right)
$$

where $\mathbf{v}$ is the pore-scale velocity, $p$ is the fluid pressure, $\rho$ is the density, $v=\mu / \rho$ is the kinematic viscosity, $\mu$ is the dynamic viscosity, $c$ is the solute concentration, and $D_{m}$ is the molecular diffusion coefficient.

The description of this problem is traditionally approached from a macroscopic point of view, by using continuum equations with parameters resulting from averaging procedures carried out on the microscopic structures of the medium (e.g., permeability, porosity) or of the flow (e.g., tortuosity, dispersivity) as explained by Whitaker [2], Berkowitz et al. [3],

\footnotetext{
*daniele.marchisio@polito.it
}

and Nordbotten et al. [4], resulting in the following continuity equation:

$$
\nabla \cdot \mathbf{V}=0
$$

in the momentum equation:

$$
\frac{\partial \mathbf{V}}{\partial t}+\mathbf{V} \cdot \nabla(\epsilon \mathbf{V})=-\frac{\nabla p}{\rho}+\mathbf{g}-\gamma \mathbf{V},
$$

and in the advection-dispersion equation:

$$
\frac{\partial(C \epsilon)}{\partial t}+\mathbf{V} \cdot \nabla C=\nabla \cdot(\epsilon \mathbf{D} \nabla C)
$$

where $\mathbf{V}$ is the Darcy velocity (or superficial velocity) and $C$ is the macroscale solute concentration (mass, volume, or number concentration of solute in the liquid or void phase) and $\epsilon$ is the porous medium porosity.

At low superficial velocities the friction coefficient is assumed to be constant: $\gamma=\mu / \rho k$, where $k$ is its permeability. In this case, if the time derivative and the inertial terms of Eq. (5) are neglected and the momentum balance equation is applied to a simple one-dimensional problem, the original phenomenological (linear) law developed by Darcy is retrieved. At higher superficial velocities, the Darcy-Forchheimer law is generally used [5-7], and the friction coefficient is assumed to be function of the fluid superficial velocity: $\gamma=\mu \epsilon / \rho \alpha+$ $\beta|\mathbf{V}|$, where now $\alpha$ and $\beta$ are the two Darcy-Forchheimer parameters. The application of the Darcy-Forchheimer law to a simple one-dimensional problem, again neglecting the time derivative and the inertial terms, results in a quadratic law, 
often written in the following form:

$$
\frac{\Delta P}{L}=150 \frac{(1-\epsilon)^{2}}{\epsilon^{3}} \frac{\mu V}{d_{\mathrm{g}}^{2}}+1.75 \frac{\rho V^{2}}{d_{\mathrm{g}}} \frac{1-\epsilon}{\epsilon^{3}},
$$

where $\Delta P$ are the pressure drops over the length $L$ of the porous medium considered and $V$ is the superficial velocity along this direction. In Eq. (7) the Darcy-Forchheimer parameters, $\alpha$ and $\beta$, are directly expressed in terms of the mean grain size characterizing the porous medium, $d_{\mathrm{g}}$, and its porosity $\epsilon$; the latter equation is known as the Ergun law.

The dispersion tensor $\mathbf{D}$ appearing in Eq. (6) is generally written as the summation of the macroscopic effective molecular diffusion $D_{0}$, and of the longitudinal, $D_{\mathrm{L}}$, and transversal, $D_{\mathrm{T}}$, dispersion coefficients [8]. The first $\left(D_{0}\right)$ is the upscaled effect of the molecular diffusion (i.e., it acts even when the fluid is at rest), while the last two are due to mechanical dispersion, caused in turn by velocity inhomogeneities at the pore scale. It is important to notice that, even if the molecular diffusion $D_{m}$ is a scalar, the macroscopic effective molecular diffusion $D_{0}$ can become a tensor when the porous medium is not perfectly isotropic. The effect of the system's heterogeneity on particle transport and the importance of diffusive processes occurring at the scale of the pores were noted in several works $[9,10]$. In general all these coefficients depend on the porosity of the porous medium, $\epsilon$, on the tortuosity (i.e., a coefficient, $\tau$, or a tensor, $\boldsymbol{\tau}$, that takes into account the geometrical complexity of the medium) and on the Péclet number of the flow (which will be defined later). The overall effect of diffusion and mechanical dispersion in the porous medium is also called "hydrodynamic dispersion."

Also for the hydrodynamic dispersion, as for the friction coefficient, laws are obtained from experimental investigations and analytical methods and expressed in terms of the relevant dimensionless numbers, as well as the relevant parameters characterizing the porous medium. However, the confidence with which these laws can be employed is not completely clear, especially due to the nonlinear dependence between the final macroscale parameters and the initial characteristics of the medium and flow properties. Moreover, the experimental determination of these parameters is often very complicated and affected by large uncertainties.

These issues motivate our research efforts in the explicit simulation of microscale (i.e., pore scale) flow and hydrodynamic dispersion with the purpose of extracting macroscale laws. In fact, pore-scale simulations can be used to confirm the theoretical and experimental correlation laws and to explicitly compute the constant parameters, usually unknown, for a specific pore geometry. Thanks to the increasing computing resources this analysis is becoming an important area of investigation (see, e.g., Refs. [11-21]), with applications in contaminant transport in aquifers, reservoir simulation, $\mathrm{CO}_{2}$ storage, colloidal transport, chromatographic separation, and catalytic reaction engineering [22], recently reviewed by Blunt et al. [23].

The objective of this work is twofold: (1) to analyze the flow and dispersion in a realistic three-dimensional geometry by solving the Navier-Stokes equation and (2) to develop and validate reliable computational tools for the efficient porescale simulation in complex geometries, accompanied by the choice of adequate numerical methods and grid convergence tests, as currently done in computational fluid dynamics (CFD). This work is moreover preparatory to the extension of the investigation of particle deposition in porous media from two-dimensional [24] to three-dimensional geometries.

The main points of this work, in comparison with other recent work are listed below. In this work the full Navier-Stokes equation is solved, instead of solving the simpler Stokes equation (often adopted in this context). Moreover, instead of building the porous medium from monodisperse spheres, in this work the porous medium has been generated by randomly packing nonspherical highly irregular nonconvex polydisperse objects, whose shape and size distribution have a direct link to experimentally characterized sands. Another important point of this work is that this operation is conducted here by using the open-source computer graphics three-dimensional code BLENDER. This code can easily overcome the limitations of other codes that are limited to the treatment of spheres and convex objects. In addition to this, particular attention is paid here to the well-known mesh generation and spatial discretization issues encountered when Eqs. (1)-(3) are solved with the finite-volume method (FVM). This is done here by using the open-source CFD code OpenFOAM. Another point of this work, beyond the fully open-source computational tools, is the usage of an "Eulerian" method to analyze solute dispersion at the pore scale. We will show that this approach, in contrast to the commonly used tracer particle "Lagrangian" approach, has many computational and practical advantages.

The article is organized as follows. First, the macroscopic problem and its classical formulation is briefly recalled and then computational tools developed to extract grain information from real images, reconstruct realistic geometries, and discretize the pore space are described. Subsequently the numerical details about the pore-scale simulations are reported together with the results; simulation predictions are eventually processed to extract velocity distributions and dispersion coefficients, as well as to assess the validity of the Fick law to describe dispersion phenomena. Conclusions and possible next steps are discussed in the last section.

\section{RELATIONSHIP BETWEEN MICROSCALE AND MACROSCALE MODELS}

The geometry considered in this work is a simple cube filled with grains or particles and immersed in a Newtonian fluid. The fluid flow has one principal direction, indicated with $x$, with fluid entering and exiting the domain from the faces orthogonal to the $x$ direction and null net fluid flow on the four faces orthogonal to the $y$ and $z$ axes. Starting from the pore-scale velocity, $\mathbf{v}(\mathbf{x})$, and the pore-scale concentration, $c(\mathbf{x})$, fields, by performing a surface average over the $y$ and $z$ directions, the macroscopic (average) Darcy velocity in the main flow direction,

$$
V_{x}(x)=\frac{\int_{0}^{\mathcal{L}_{y}} \int_{0}^{\mathcal{L}_{z}} v_{x}(\mathbf{x}) \chi(\mathbf{x}) d y d z}{\mathcal{L}_{y} \mathcal{L}_{z}}
$$


and the macroscopic (average) solute concentration,

$C(x)=\frac{\int_{0}^{\mathcal{L}_{y}} \int_{0}^{\mathcal{L}_{z}} c(\mathbf{x}) \chi(\mathbf{x}) d y d z}{\int_{0}^{\mathcal{L}_{y}} \int_{0}^{\mathcal{L}_{z}} \chi(\mathbf{x}) d y d z}=\frac{\int_{0}^{\mathcal{L}_{y}} \int_{0}^{\mathcal{L}_{z}} c(\mathbf{x}) \chi(\mathbf{x}) d y d z}{\epsilon(x) \mathcal{L}_{y} \mathcal{L}_{z}}$,

are readily defined, where $\chi$ is the indicator function of void space, $\epsilon(x)$ is the porosity of the sections, $\mathcal{L}_{x}, \mathcal{L}_{y}, \mathcal{L}_{z}$ are the extensions of the domain in the $x, y$, and $z$ directions, respectively, and $v_{x}$ is the $x$ component of $\mathbf{v}(\mathbf{x})$. The macroscopic continuity equation [Eq. (4)] becomes the condition of constant macroscopic superficial velocity (or constant mass flux) along the $x$ direction [25-27]. Based on these definitions one can calculate the deviations of velocity, $v_{x}^{\prime}=v_{x}-V_{x} / \epsilon$, and concentration, $c^{\prime}=c-C$, from their average values, and obtain the following macroscopic equation:

$$
\frac{\partial(C \epsilon)}{\partial t}+V_{x} \frac{\partial C}{\partial x}=\frac{\partial}{\partial x}\left(\epsilon D \frac{\partial C}{\partial x}\right)
$$

where the other advection terms are null due to the incompressibility condition and the boundary conditions of zero net flux along the $y$ and $z$ directions employed in this work. These boundary conditions also simplify the second-order dispersion tensor $\mathbf{D}$ of Eq. (6), which results in a scalar equal to the summation of the already introduced effective molecular and longitudinal dispersion coefficients:

$$
\left(\epsilon D_{0} \frac{\partial C}{\partial x}-\epsilon\left\langle v_{x}^{\prime} c^{\prime}\right\rangle\right)=\epsilon D \frac{\partial C}{\partial x}, \quad \text { with } \quad D=D_{0}+D_{\mathrm{L}},
$$

where for the unclosed term $\left\langle v_{x}^{\prime} c^{\prime}\right\rangle$ the standard Fick's law was implicitly used:

$$
\left\langle v_{x}^{\prime} c^{\prime}\right\rangle=\frac{1}{\epsilon \mathcal{L}_{y} \mathcal{L}_{z}} \int_{0}^{\mathcal{L}_{y}} \int_{0}^{\mathcal{L}_{z}} v_{x}^{\prime} c^{\prime} d y d z \approx-D_{\mathrm{L}} \frac{\partial C}{\partial x} .
$$

For a detailed derivation of the effective molecular diffusion $D_{0}$ and its relation to the microscale molecular diffusion $D_{m}$, we refer to classical important works [2,25,28-30]. When the porosity $\epsilon$ is constant in time, one can rewrite Eq. (10) as follows:

$$
\frac{\partial C}{\partial t}+\left(\frac{V_{x}}{\epsilon}-\frac{1}{\epsilon} \frac{\partial \epsilon D}{\partial x}\right) \frac{\partial C}{\partial x}=D \frac{\partial^{2} C}{\partial x^{2}},
$$

where $V_{\mathrm{eff}}=\left(\frac{V_{x}}{\epsilon}-\frac{1}{\epsilon} \frac{\partial \epsilon D}{\partial x}\right)$ takes the role of the effective velocity. The Fickian hypothesis of Eq. (12) can be invalid for more complex porous structures characterized by low porosity, fractures, and multiscale porosity [19,31]. In these cases, many different models have been proposed such as the fractional advection-diffusion reaction [32,33] or the continuous-time random walk [34]. Both are based on the generalization of Brownian motion, considering Levy flight processes, while Tartakovsky et al. [35] proposed to introduce a stochastic perturbation in the Darcy equation. For multiscale porosity media, an extension of Eq. (6) called the multiple-rate mass transfer model [36], or the similar mobile-immobile (or dual porosity) model [37], can be used. These models assume the existence of an additional phase and an associated pore space with a different velocity and volume fraction. We refer to the specialized literature cited for more details about these complex models.
Different relationships can be used to estimate $D$ as a function of the Péclet number: $\mathrm{Pe}=V_{x} d_{\mathrm{g}} / \epsilon D_{\mathrm{m}}$. For $\mathrm{Pe}$ tending to zero the following relationship holds [25]:

$$
\frac{D}{D_{m}}=\frac{1}{\tau^{2}}
$$

where $\tau$ is the tortuosity of the porous medium. Many definitions and formula have been proposed, and recent works [38-40] have demonstrated their effectiveness and the difference between formulations. The most common definition involves the computations of streak lines (i.e., Lagrangian trajectories) of the flow and the calculation of the ratio between their length and the porous medium size. Koponen et al. [38] and Duda et al. [40] proposed a simpler way to compute $\tau$ as a volume integral:

$$
\tau=\frac{\int|\mathbf{v}| d \mathbf{x}}{\int v_{x} d \mathbf{x}} .
$$

A widely used correlation for the hydrodynamic dispersion at higher Pe numbers is $D=\alpha V_{x}$, where $\alpha$ is of the order of the mean grain size. van Milligen and Bons [41] derived a single equation valid for different ranges of Péclet numbers:

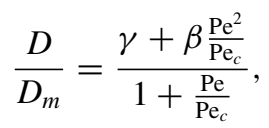

where $\mathrm{Pe}_{c}$ is the critical Pe number, $\gamma$ is the coefficient that scales the macroscale dispersion, $D$, to the molecular diffusivity, $D_{m}$, for Pe $\ll 1$, whereas $\beta$ is the linear coefficient between $D, D_{m}$, and Pe for Pe $\gg 1$. The three asymptotic regimes can be identified by defining $\delta=\sqrt{\frac{\gamma}{\beta}}$ :

$$
D \approx\left\{\begin{array}{l}
\gamma D_{m} \quad \text { when } \mathrm{Pe} \ll \delta \sqrt{\mathrm{Pe}_{c}} \\
\beta \mathrm{Pe}^{2} / \mathrm{Pe}_{c} \quad \text { when } \delta \sqrt{\mathrm{Pe}_{c}} \ll \mathrm{Pe} \ll \mathrm{Pe}_{c} \\
\beta \mathrm{Pe} \quad \text { when } \mathrm{Pe} \gg \mathrm{Pe}_{c} .
\end{array}\right.
$$

This simple relationship is not universally valid; in fact, it typically does not hold for more complex porous media (where, for example, a nonlinear relation can appear for high Péclet number) and in the preasymptotic regime. Another common expression is the correlation proposed by Bear [25]:

$$
\frac{D}{D_{m}}=\gamma+\frac{\alpha V}{D_{m}} \frac{\mathrm{Pe}}{\mathrm{Pe}+2+4 \delta^{2}},
$$

where $\gamma$ and $\delta$ have the same physical meaning of the coefficients appearing in Eq. (17).

\section{THREE-DIMENSIONAL GRAIN PACKING AND MESH GENERATION}

In this section the strategy employed to create the threedimensional porous medium used in this work and the mesh generation procedure for performing the finite-volume discretization are briefly described.

\section{A. Grain packing generation}

The first necessary step requires building a representative microscopic model of a porous medium sample in order to simulate fluid flow in these systems. There are many ways 
to obtain such a model: the first choice is between real sand sample images, for example, experimentally acquired by microcomputer tomography techniques, and realistic reconstructions by means of suitable algorithms [23]. Although the former provides representations of the pore microstructure which are real, the extraction procedures (segmentation, surface reconstruction, etc.) are usually quite complicated, expensive, and with a high degree of arbitrariness. Therefore, it was chosen to reconstruct a realistic porous medium with ad hoc algorithms. It has to be noted that when following this approach one has to make sure that the characteristics and features of the generated porous medium (e.g., porosity, grain size distribution, and pore-throat structure) are representative of a real system. Notwithstanding these difficulties, there are several advantages with this approach; it is possible, for example, to test simple geometries (where real grains are modeled as spheres) that make it easier to study multiple test cases (e.g., models with different grain diameter or porosity) in a semiautomatic fashion [24].

A number of methods have been proposed to synthetically generate realistic packed geometries. Some of them are based on the idea of representing the medium with a pore-network model, whereas some others "reconstruct" the entire structure of the porous medium, down to the single grains, using different algorithms (i.e., random sequential adsorption, gravitational deposition, collective arrangement, binary random fields) [42-47]. In this work, two different methods of packing generation, both based on the open-source library Bullet Physics [48], were tested: SETTLEDYN [49] and BLENDER [50]. The tested methods simulate the sedimentation of real three-dimensional grains, represented by convex polygonal surface meshes, generating loose sandlike structures from given particle forms and grain size distributions.

The first step is the definition of grain shape and grain size distribution, which in this work were obtained, respectively, by two-dimensional scanning electron microscopy (SEM) scans (see, for example, Fig. 1) and static-light scattering measurements carried out on standard sand samples. For the

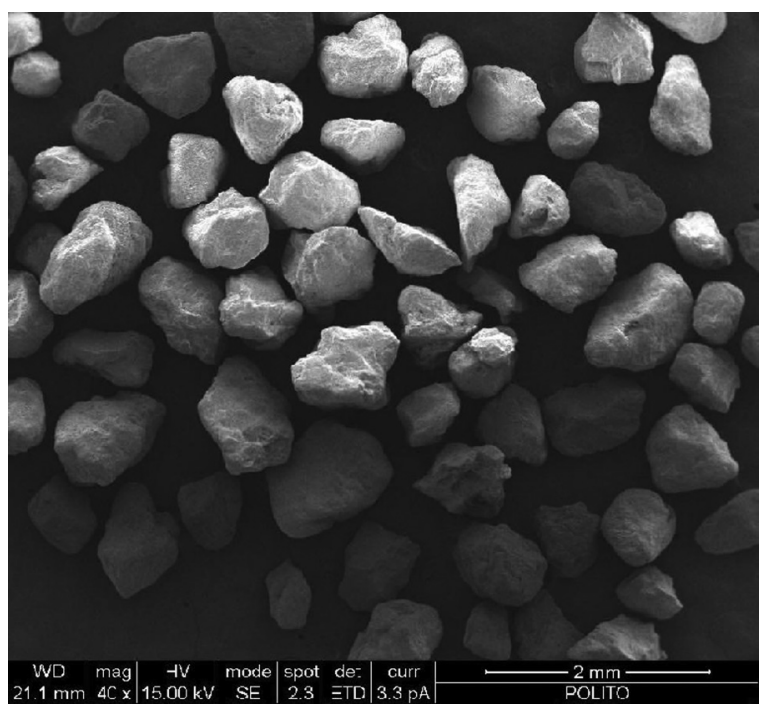

FIG. 1. SEM image of the real sand sample used to extract grain size distribution and approximate the grain shapes.

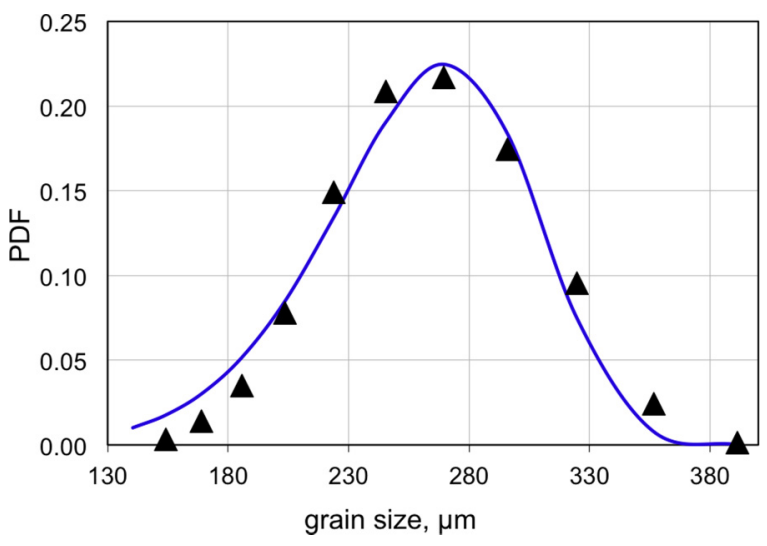

FIG. 2. (Color online) Grain size distribution of the sand packing under study (filled triangles) and approximation with Weibull distribution (continuous blue line).

case investigated in this work the final mean grain size $d_{50}$ was equal to $0.277 \mathrm{~mm}$. The grain size distribution was then fitted with a Weibull distribution [6,24], resulting in a shape parameter equal to seven, and was then randomly sampled when creating the packing. Figure 2 compares the experimentally measured grain size distribution of the sample with the reconstructed Weibull distribution.

Different confined packings of thousands of grains were then generated by randomly sampling the Weibull distribution, on a single core machine, with a large quantity of memory in a few hours. These tests highlighted, for the operating conditions investigated in this work, a higher flexibility when using BLENDER, especially in terms of robustness and convergence. One of these packings (created with BLENDER) with more than 3000 grains was extensively used in this work for most of the pore-scale simulations. The final optimized porous medium is a cube domain of $2.1 \mathrm{~mm}$ length and is characterized by an average porosity of 0.35 . By splitting the geometry in 10 subvolumes along the $x$ axis, it was noted that the porosity (in these subvolumes) fluctuates around this value by approximately $1 \%$, while the porosity calculated on the surfaces varies between 0.31 and 0.39 .

\section{B. Mesh generation}

After the generation of the grain packing, the geometric representation (made of polygonal surface mesh) of the resulting grain packings was cleaned through mesh resampling, in order to remove intersections and artifacts. This was done with the marching cubes algorithm [51,52]. An example is reported in Fig. 3, which shows a surface mesh, obtained with the algorithms previously described and grains characterized by very sharp edges, and the final resampled mesh. After resampling the pore domain is ready to be discretized through body-fitted meshes, as commonly done when the FVM is used.

As mentioned before, great care was put forward during the preprocessing phase of this work in order to ensure the highest possible accuracy with respect to numerical and computational issues. A careful analysis of the mesh generation process now follows, along with the description of the steps taken to ascertain the validity of the final mesh thus obtained. 

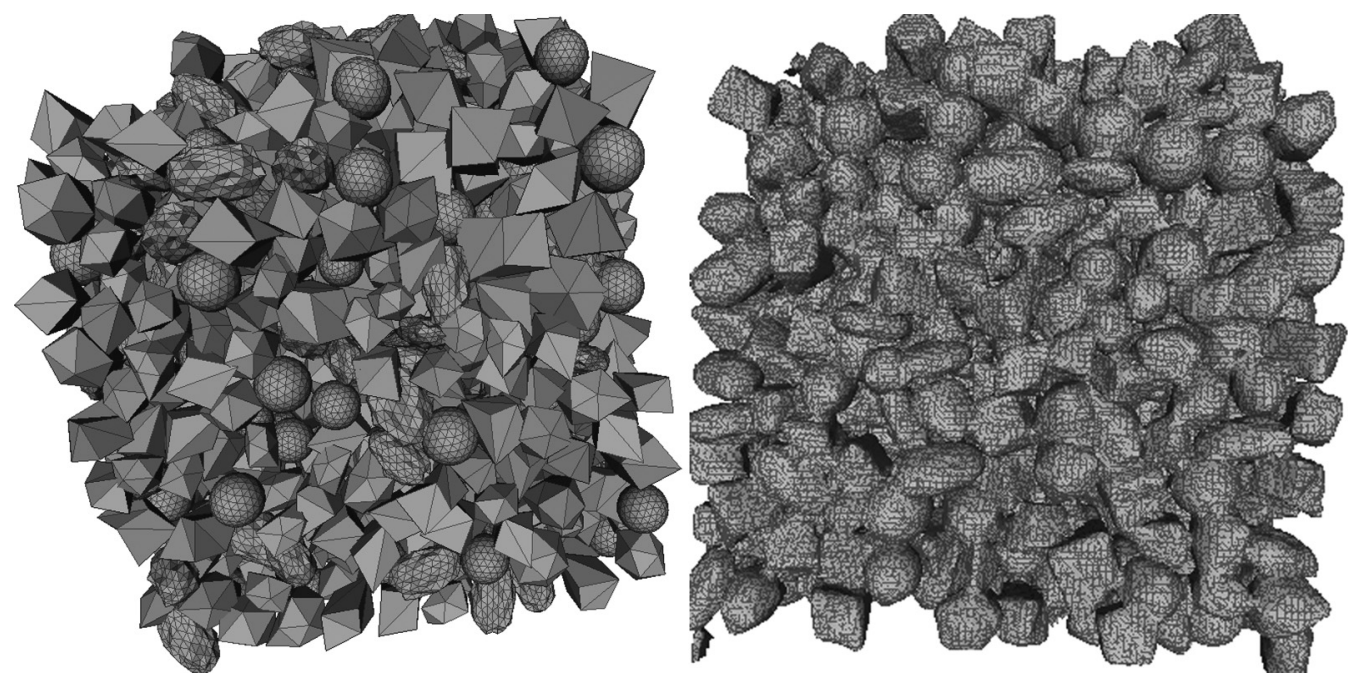

FIG. 3. Mesh resampling, randomization, and regularization. Original surface mesh (left) and resampled mesh (right).

The mesh utility snappyHexMesh, native to the opensource package OpenFOAM [53], was used. Grid building was performed in two steps. First, a structured, Cartesian mesh was created in the fluid portion of the domain, in order to minimize average nonorthogonality and skewness in the final mesh. The handling of the actual surface of the solid part in this initial step is done in a way similar to how immersed boundary meshes are constructed, resulting in a stepwise approximation of the grains. However, a precise reconstruction of the actual grain geometry was deemed essential in this work, thus requiring a second step in which the mesh was modified by means of relocating boundary vertices, resulting in a body-fitted mesh. A visual aid for the description of this process is found in Fig. 4.

In addition to these two steps, one of the subsequent grid refinement steps is also visualized. In fact, the most critical operation was the construction of a mesh of high quality to ensure grid-independent results. To this end, a number of refinements were performed and two parameters monitored throughout in order to quantitatively assess the resulting mesh suitability for use with the CFD code. Given the heavy computational resources required for the CFD calculations (as described in the following sections), these tests were not performed on the full domain used for the production runs, but rather on two smaller subvolumes. The linear dimensions of each of these volumes are smaller by a factor of four with respect to the complete geometry, resulting in $500 \mu \mathrm{m} \times 500$ $\mu \mathrm{m} \times 500 \mu \mathrm{m}$ cubes. This is shown in Fig. 5 .

An outline of all the mesh modification steps, along with the calculated relevant parameters associated with each test (for one of the two subvolumes), is reported in Table I. As a qualitative description of these cases, the first represents a coarse grid, whereas the second is obtained after a uniform refinement across the computational domain. The following cases (near-grain ref. 1, 2, 3, and 4) differ by representing progressive refinements of mesh cells size near the border of the grains, with this region being chosen in order to increase the precision in the description of the momentum and particle concentration boundary layers, where the gradients of these properties will be stronger. The first of the two properties of interest being monitored at this stage is the porosity: as can be seen from Table I its value changes (increasing slightly) with the increase in the number of cells. This is due to the addition of more cells near the surface of the grains allowing not only for a better numerical solution, but also for a more precise reconstruction of the actual porous medium geometry. Again, Fig. 4 exemplifies this process. The fourth case (near-grain ref. 2 ) reaches a satisfactory description in such respect. The other parameter needed to assess the grid independence of the results for the flow field is the equivalent diameter $d_{\mathrm{g}}^{*}$. Following the
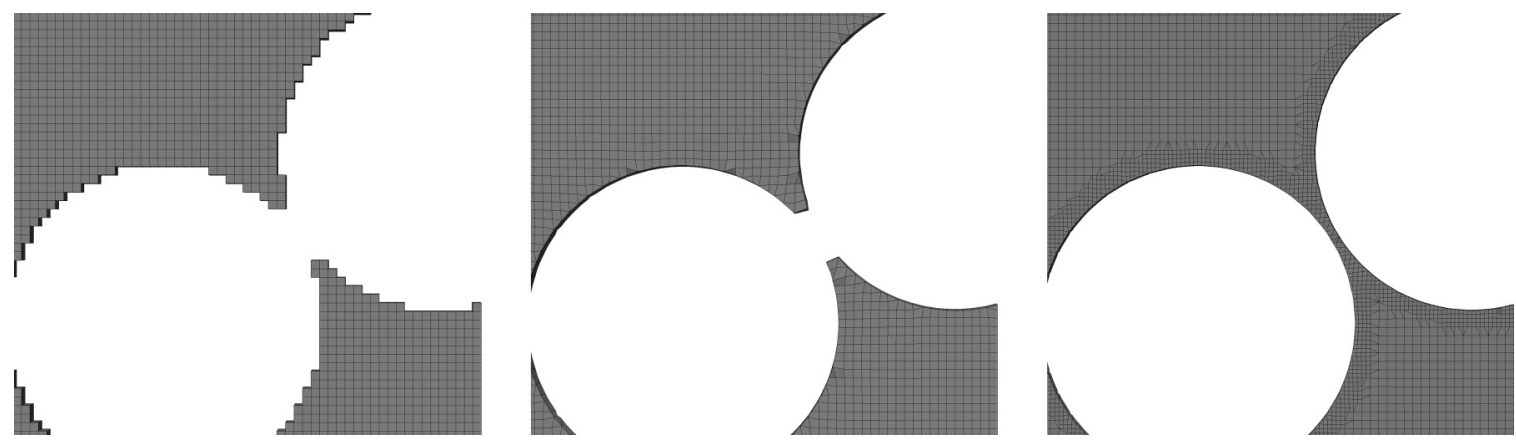

FIG. 4. Details of the mesh refinement process. Stepwise castellation (left), body fitting via vertices moving (center), and precise geometry reconstruction with subsequent mesh refinements (right). 


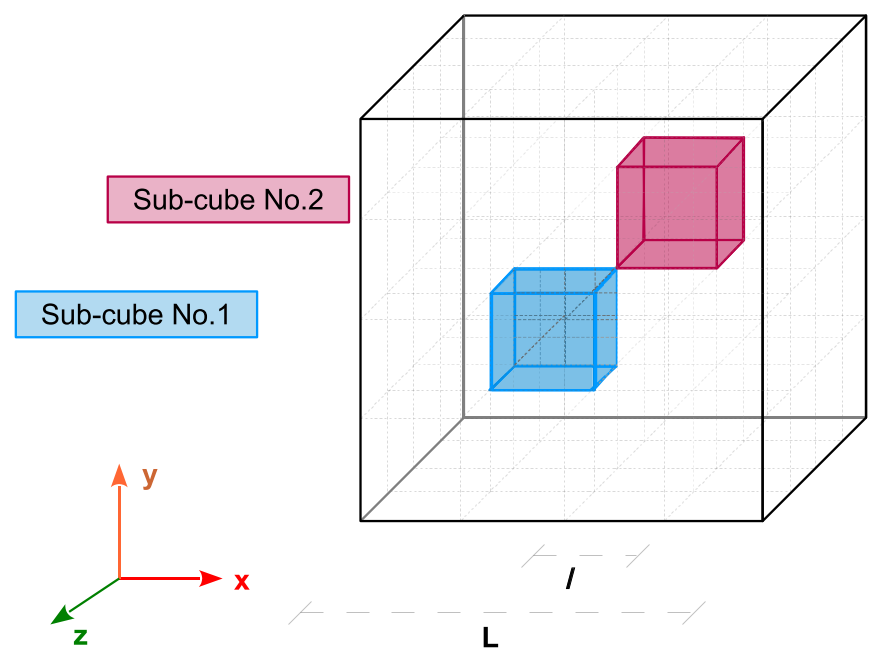

FIG. 5. (Color online) Complete geometry bounding box and location of the two subvolumes chosen for the grid independence analysis.

same methodology applied in a previous work (and described in detail therein [24]), for each level of grid refinement steadystate flow field simulations at different superficial velocities were run and the pressure drop results compared with the predictions of the Ergun law for that system [see Eq. (7)]. Treating the grain diameter as a fitting parameter, the effective diameter $d_{\mathrm{g}}^{*}$ reported in Table I results from the closest fitting of the obtained CFD results with the theoretical predictions. Considering the variation of $d_{\mathrm{g}}^{*}$ with increasing mesh cells number, again the fourth case provides a good resolution of the momentum boundary layer, as the heavy mesh refinements performed in the subsequent cases correspond to negligible changes in effective grain diameter. Thus, the mesh for the complete geometry used in the final runs, from which all the results presented in this work were obtained, was built in such a way to result in the same features as those identified in the case (near-grain ref. 2) of Table I.

\section{NUMERICAL DETAILS OF THE CFD SIMULATIONS}

The flow field in the reconstructed geometry was simulated with the open-source code OpenFOAM. The three-dimensional incompressible steady Navier-Stokes equations were solved with the simpleFoam solver. As mentioned the equations were discretized with the FVM. The numerical schemes used were chosen in order to minimize the numerical errors for the

TABLE I. Number of mesh cells, porosity, and effective grain diameter $(\mu \mathrm{m})$ for each of the grid refinement steps.

\begin{tabular}{lccc}
\hline \hline Case & Mesh cells (thousands) & $\epsilon(-)$ & $d_{\mathrm{g}}^{*}(\mu \mathrm{m})$ \\
\hline Coarse grid & 37 & 0.3025 & 203.0 \\
Unif. ref. & 308 & 0.3088 & 207.8 \\
Near-grain ref. 1 & 1.218 & 0.3102 & 217.4 \\
Near-grain ref. 2 & 2.284 & 0.3104 & 214.9 \\
Near-grain ref. 3 & 3.539 & 0.3105 & 216.1 \\
Near-grain ref. 4 & 5.053 & 0.3105 & 215.6 \\
\hline \hline
\end{tabular}

different test cases characterized by a wide range of Reynolds numbers (see Table II). A second-order central scheme with limiters to avoid oscillations was used for spatial discretization, and the SIMPLEC scheme was used to overcome the pressurevelocity coupling problem.

The whole cubic domain, extracted by the packing and reported in Figs. 3 and 5, was studied with a fixed hydraulic head drop between inlet and outlet and with symmetric conditions on the lateral boundaries. On these boundaries it is imposed that the derivatives of the velocity components along the orthogonal direction are null and that the orthogonal velocity component is also null. For example, for the two boundaries orthogonal to the $y$ axis, $V_{y}=0$, whereas $V_{x}$ and $V_{z}$ are kept free; the same conditions hold also for the boundaries orthogonal to the $z$ axis. This means that the resulting main flow is directed along the $x$ axis, and there is no flow escaping from lateral boundaries (in the $y$ and $z$ directions). The problem of the best boundary conditions was also investigated in our previous work, where it was shown that this set of conditions resulted in the least invasive approach, which could avoid the use of pre- and postmixing conditions, as well as the exclusion of the external part of the flow from the statistical analysis [6,24]. The simulated domain length was $\mathcal{L}_{x}=\mathcal{L}_{y}=\mathcal{L}_{z}=2 \mathrm{~mm}$, and it contained about 3000 sand grains. For each value of hydraulic head drop, the mean flow rate and Darcy fluxes $V_{x}$ were calculated in sections of the porous medium orthogonal to the mean flow field. A summary of the resulting Reynolds, $\operatorname{Re}=d_{50} V_{x} / \epsilon \nu$, and Péclet numbers, $\mathrm{Pe}=d_{50} V_{x} / \epsilon D_{m}$, is reported in Table II; both dimensionless numbers are evaluated by using $d_{50}=$ $0.270 \mathrm{~mm}$ as an estimation of the grain size $d_{\mathrm{g}}$. As is seen, the flow fields obtained at different Reynolds numbers were used to transport an Eulerian solute concentration field with different molecular diffusion coefficients resulting in Péclet numbers ranging from $10^{-2}$ up to $10^{6}$.

The simulations of the flow field were carried out in parallel on a Linux workstation with 12 Intel Xeon X6960 cores. The computational bottleneck for these simulations turned out to be the huge memory usage (100 GB), rather than the convergence iterations (usually less than 100). In our configuration this resulted in $12 \mathrm{hr}$ of total CPU time for each flow field computation.

The simulations of solute transport were carried out in OpenFOAM but with a unsteady solver, up to the full saturation of the medium. A constant concentration (constant Dirichlet boundary conditions with a fixed concentration equal to unity) was employed as boundary condition at the inlet. A null concentration gradient was instead used as boundary condition on the grain surfaces (neglecting therefore particle deposition) and on the lateral and outlet boundaries (approximating an infinite medium).

It is worth mentioning here that the grid independence study conducted in the previous section should be repeated also for the solute dispersion simulations, in order to ensure that not only the predicted flow field is grid independent, but also that grid independence holds for the solute concentration field. A computational grid resulting in a grid-independent flow field may not result in a grid-independent solute concentration field, because the solute concentration gradients occur on length scales smaller than the those of momentum, 
TABLE II. Superficial flow velocities, $V_{x}$, Reynolds numbers, Re, molecular diffusion coefficients, $D_{m}$, and resulting Péclet numbers, Pe.

\begin{tabular}{|c|c|c|c|c|}
\hline \multirow[b]{2}{*}{$V_{x}\left(\mathrm{~m} \mathrm{~s}^{-1}\right)$} & \multirow[b]{2}{*}{$\operatorname{Re}$} & \multicolumn{3}{|c|}{$\mathrm{Pe}$} \\
\hline & & $D_{m}=5 \times 10^{-11}\left(\mathrm{~m}^{2} \mathrm{~s}^{-1}\right)$ & $D_{m}=5 \times 10^{-10}\left(\mathrm{~m}^{2} \mathrm{~s}^{-1}\right)$ & $D_{m}=5 \times 10^{-9}\left(\mathrm{~m}^{2} \mathrm{~s}^{-1}\right)$ \\
\hline $1.2 \times 10^{-7}$ & $9.6 \times 10^{-5}$ & $1.9 \times 10^{-2}$ & $1.9 \times 10^{-1}$ & $1.9 \times 10^{0}$ \\
\hline $1.2 \times 10^{-6}$ & $9.6 \times 10^{-4}$ & $1.9 \times 10^{-1}$ & $1.9 \times 10^{0}$ & $1.9 \times 10^{1}$ \\
\hline $1.2 \times 10^{-5}$ & $9.6 \times 10^{-3}$ & $1.9 \times 10^{0}$ & $1.9 \times 10^{1}$ & $1.9 \times 10^{2}$ \\
\hline $1.2 \times 10^{-4}$ & $9.6 \times 10^{-2}$ & $1.9 \times 10^{1}$ & $1.9 \times 10^{2}$ & $1.9 \times 10^{3}$ \\
\hline $1.2 \times 10^{-3}$ & $9.6 \times 10^{-1}$ & $1.9 \times 10^{2}$ & $1.9 \times 10^{3}$ & $1.9 \times 10^{4}$ \\
\hline $1.2 \times 10^{-2}$ & 9.2 & $1.8 \times 10^{3}$ & $1.8 \times 10^{4}$ & $1.8 \times 10^{5}$ \\
\hline $8.3 \times 10^{-2}$ & 66 & $1.3 \times 10^{4}$ & $1.3 \times 10^{5}$ & $1.3 \times 10^{6}$ \\
\hline $3.9 \times 10^{-1}$ & 314 & $6.1 \times 10^{4}$ & $6.1 \times 10^{5}$ & $6.1 \times 10^{6}$ \\
\hline
\end{tabular}

when the Schmidt number, Sc, is greater than one. Although the computational grids should be refined accordingly, the grid used to obtained a grid-independent flow field oftentimes results in a grid-independent solute concentration field. This condition will certainly hold here, where there is not solute concentration boundary layer to resolve, as the solute is not reacting on the grain surface, but is simply advected and diffused, and therefore gradients at the pore scale are not very large.

These more computationally demanding simulations were carried out in parallel on the Curie supercomputer, owned by GENCI and operated by CEA, using 48 Intel Nehalem-EX $\mathrm{X} 7560$ cores. In our configuration this resulted in $240 \mathrm{hr}$ of total CPU time for each scalar transport computation. The domain was decomposed by simple splitting the domain in each direction by powers of two. Scalability tests were performed for the flow solver and for the scalar transport, showing that,

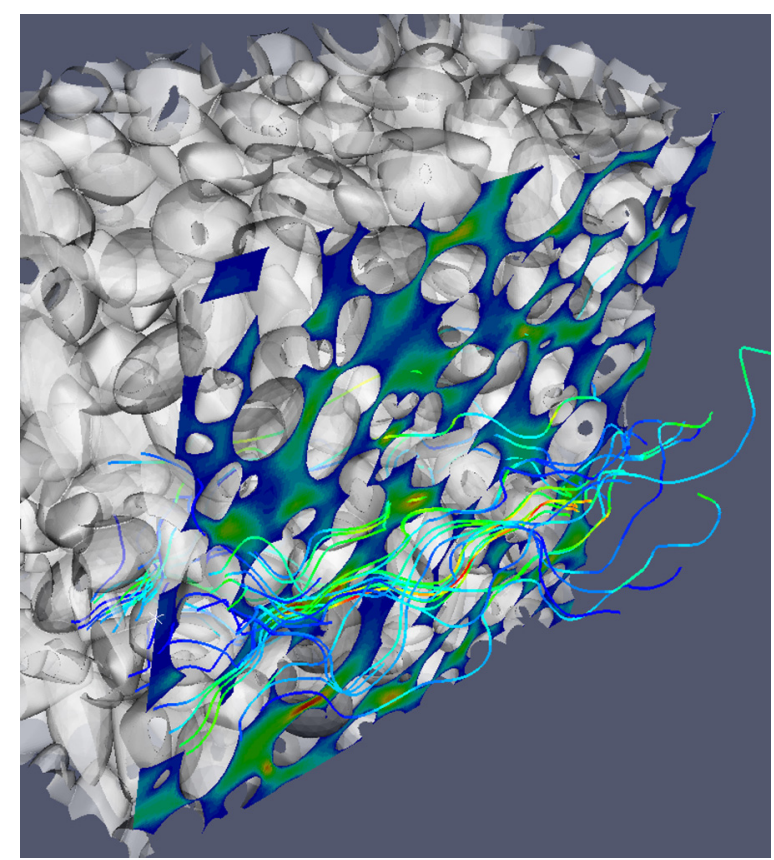

FIG. 6. (Color online) Visualization of the flow field in the porous medium for $\operatorname{Re}=9.6 \times 10^{-3}$. Velocity magnitude is computed in a central slice, and some flow streamlines are shown. Color coding from blue to red: $0-2.5 \times 10^{-6}$. even if the three-dimensional mesh is highly irregular and unstructured, it is possible to reach an almost linear speed-up up to 512 cores, excluding the I/O operations. Due to the high Péclet number flows a second-order limited upwind space discretization with second-order Crank-Nicholson time stepping was used to solve the solute concentration transport equation [i.e., Eq. (3)].

\section{RESULTS AND DISCUSSION}

In what follows the results of our simulations are presented, focusing first on the flow field predictions, and subsequently on the solute transport predictions.

\section{A. Fluid flow}

Figure 6 reports a typical example of the flow field inside the porous medium for a Reynolds number of $9.6 \times 10^{-3}$. We begin our analysis by plotting the normalized pressure drops, $\Delta p / \rho V_{x}^{2} \mathcal{L}_{x}$, versus the normalized Darcy velocity, $V_{x} / \nu$, resulting in the well-known behavior reported in Fig. 7. As expected two domains are identified, one at low Darcy flux values corresponding to the region of validity of the Darcy law (and a linear behavior in this graph), and a second one at higher values of Darcy flux where this law is not valid anymore. From the slope of the curve (in the first linear region) an intrinsic permeability of $4.0 \times 10^{-8} \mathrm{~cm}^{2}$ can be obtained. By using

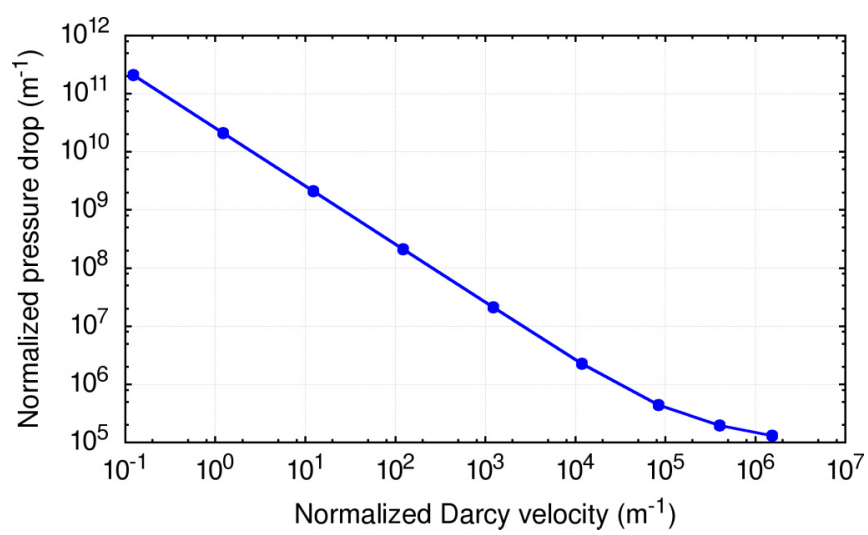

FIG. 7. (Color online) Normalized pressure drops, $\Delta p / \rho V_{x}^{2} \mathcal{L}_{x}$, bs normalized superficial velocity, $V_{x} / \nu$. The slope of the first part of the curve represent the inverse of the permeability of the medium. 

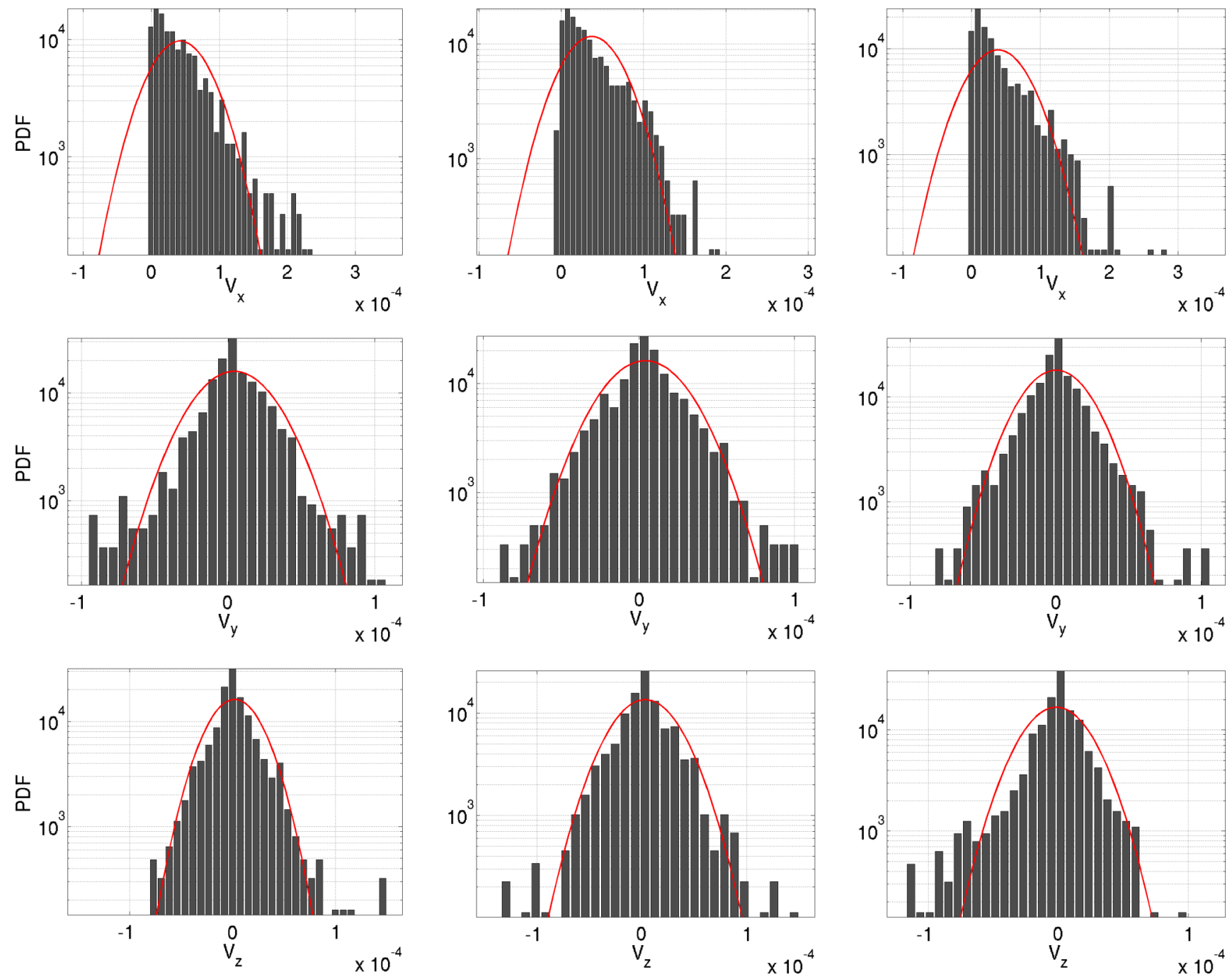

FIG. 8. (Color online) Probability density functions $\left(\mathrm{m}^{-1} \mathrm{~s}\right)$ for $\mathrm{Re}=9.6 \times 10^{-3}$ and for, from top to bottom, the $x$-, $y$-, and $z$-velocity components $\left(\mathrm{m} \mathrm{s}^{-1}\right)$ and for, from left to right, the first, fourth, and tenth section. The histogram corresponds to the actual distribution as calculated from the simulations, whereas the red curve is the Gaussian distribution with identical mean and variance.

Eq. (15), the tortuosity, $\tau$, was estimated in our case to be equal to 1.2 for low Re. However, for Reynolds numbers greater than 10 it increases and reaches a value of 1.7 for $\mathrm{Re}=314$. This is due to the different flow conditions in the pore space arising at higher velocities, changing the trajectories of the moving fluid and thus $\tau$.

Further analysis of the simulation data requires some upscaling, for which different methodologies were proposed, and we refer to the work of Cushman et al. [54] for an overview. In the present work simulation results were analyzed by both volume-averaging on the whole three-dimensional domain and by surface-averaging on 10 equispaced sections orthogonal to the main flow direction $x$. In addition to that these 10 surfaces were also used to evaluate how relevant properties are spatially distributed.

For example, by analyzing how the fluid velocity differs from point to point on these 10 surfaces, the data reported in Figs. 8 and 9 were obtained. These figures show the velocity distributions for the $x, y$, and $z$ fluid velocity components, in three of the 10 sections investigated, for two superficial velocities, resulting in Reynolds numbers of $\mathrm{Re}=9.6 \times 10^{-3}$ and of 66, respectively. The histograms were computed with 1000 samples on each surface and are reported in logarithmic scale. It is worth mentioning that excluding from the analysis the external portion of the flow (to reduce the possible effects of boundary conditions) does not significantly change the results. As is possible to see from Fig. 8, the velocity distribution for the $x$ component has a maximum corresponding to the superficial velocity, $V_{x}$, but highlights in the section regions where the fluid moves with different velocities. Comparison of these distributions with the equivalent Gaussian distributions (that share the same mean and variance) highlights a much higher frequency of velocities close to the mean value and, at the same time, the existence of larger tails of the distributions. Closer observation of the distributions in the $y$ and $z$ directions leads to similar conclusions, with the only difference that now these distributions are centered on zero (as there is no net flux in these directions) and exhibit a certain symmetry and a shape slightly closer to that of a Gaussian distribution. When the superficial velocity is increased the situation observed is very similar (see 

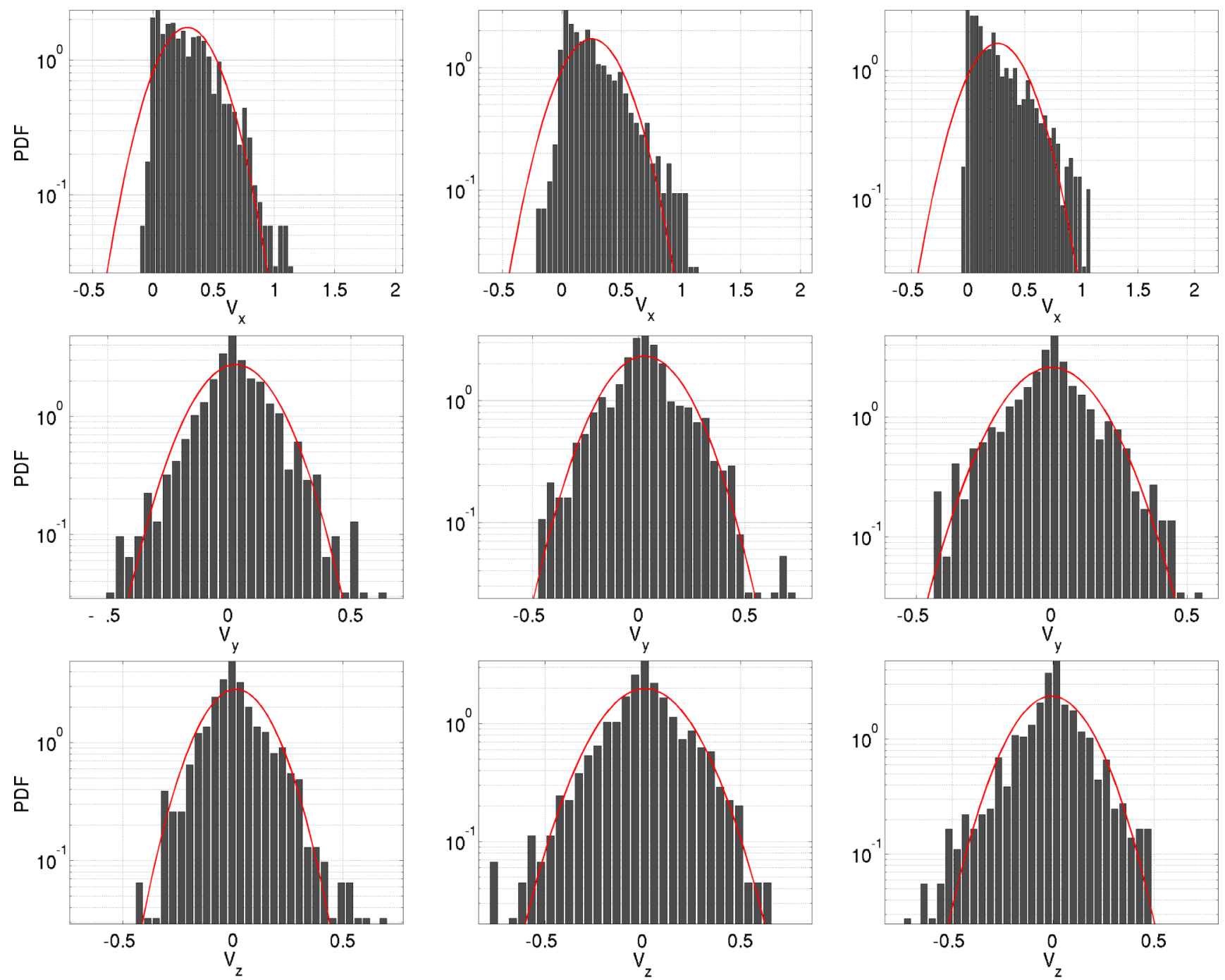

FIG. 9. (Color online) Probability density functions $\left(\mathrm{m}^{-1} \mathrm{~s}\right)$ for $\mathrm{Re}=66$ and for, from top to bottom, the $x$-, $y$-, and $z$-velocity components $\left(\mathrm{m} \mathrm{s}^{-1}\right)$ and for, from left to right, the first, fourth, and tenth section. The histogram corresponds to the actual distribution as calculated from the simulations, whereas the red curve is the Gaussian distribution with identical mean and variance.

Fig. 9) to the previous one, as in this case the distributions are characterized by larger variances. Comparison of Fig. 8 with Fig. 9 also highlights that when Re is low, the distributions are very skewed and almost every fluid element in the section moves with $V_{x}>0$. However, when $\mathrm{Re}=66$, noticeable fractions of the section show negative values for $V_{x}$; this is an indication of high-Re circulation inside the porous medium, as also indicated by other authors [55-57].
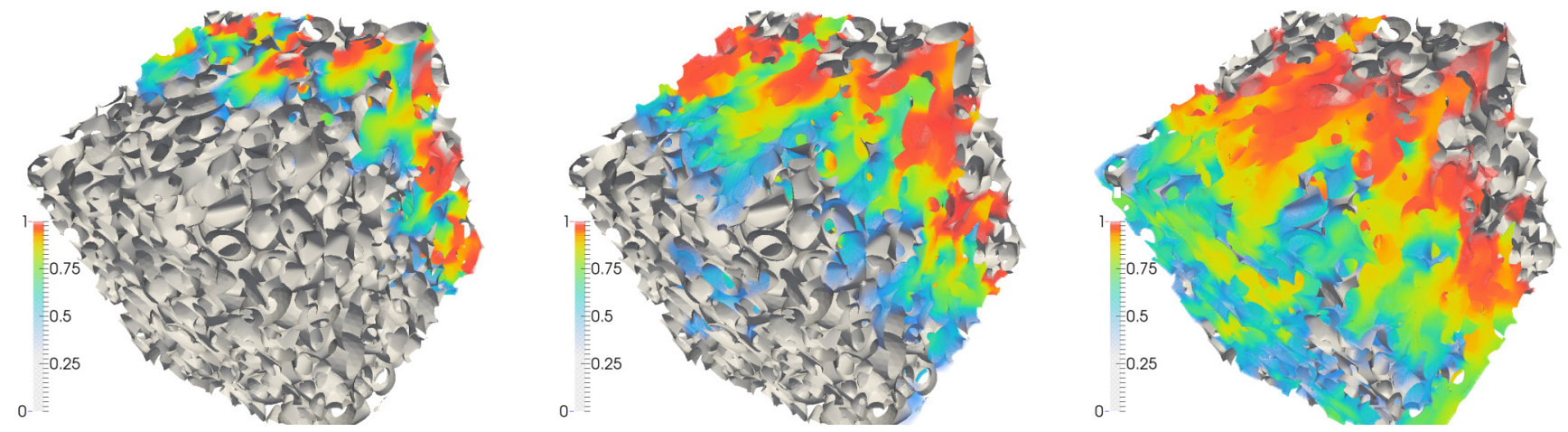

FIG. 10. (Color online) Contour plots of the solute dimensionless concentration in the porous medium at three instants of the simulation for $\mathrm{Pe}=1.9 \times 10^{3}$. 


\section{B. Hydrodynamic dispersion}

To quantify the effect of these distributions in generating dispersion phenomena in the porous medium, let us analyze the results of the solution dispersion simulations. One typical result is reported in Fig. 10, where the normalized solute concentration at three instants of the simulation is reported. As is seen, as the solute moves downstream hydraulic dispersion takes place, and the concentration front becomes more and more smoothed out.

As already said, the three-dimensional results were surfaceaveraged on sections perpendicular to the main flow direction, and, among other variables, the average solute concentration in these sections was tracked versus time. The resulting time-evolution curves (known as breakthrough curves) were then used to characterize the dispersion dynamics in the medium. Three typical results for different Re and Pe numbers are reported in Fig. 11, for nine equidistant sections, from $x=0.15 \mathcal{L}_{x}$ to $x=0.95 \mathcal{L}_{x}$ (to avoid boundary effects).

The breakthrough curves reported in Fig. 11 with continuous lines correspond to three different regimes. In the first one (top) molecular diffusion is prevailing over advective transport, in the second and third ones (middle) the two effects are of the same order of magnitude, while in the fourth one (bottom) molecular diffusion can be neglected with respect to advection. Due to the small changes in porosity of the sections, some of the curves of contiguous sections overlap.

The concentration transport at the macroscale (as sketched in Fig. 11) should obey the macroscopic advection-diffusion equation reported in its general form in Eq. (6), and for the problem investigated in this work, Eq. (6) takes the one-dimensional form reported in Eq. (13), whose coefficients are in turn unknown. Neglecting the presence of a possible stagnant phase or a retardation factor, the only actual unknown parameter is the hydrodynamic dispersion. However, also the effective transport velocity $\left(V_{\text {eff }}\right)$ was included as a unknown parameter, to check the validity of the transport equation. Since we are considering a volume comparable to the representative elementary volume, there are not enough data along the $x$ direction, but temporal data can be indeed considered (for fixed spatial locations).

The two unknown parameters (i.e., $V_{\text {eff }}$ and $D$ ) were computed with two methods. The first method is based on the inverse problem formulation. Given the breakthrough curves (concentration over time), the effective parameters are estimated with nonlinear least square minimization using standard optimization techniques. The second strategy is instead based on the method of moments [58], where the unknown parameters are calculated from the first three normalized centered temporal moments at the outlet of the computational domain:

$$
\begin{aligned}
& M_{0}=\int_{0}^{\infty} g\left(x=\mathcal{L}_{x}, t\right) d t ; \quad M_{1}=\int_{0}^{\infty} \frac{g\left(x=\mathcal{L}_{x}, t\right) t}{M_{0}} d t \\
& M_{2}=\int_{0}^{\infty} \frac{g\left(x=\mathcal{L}_{x}, t\right) t^{2} d t}{M_{0}}-M_{1}^{2}
\end{aligned}
$$

where $g(x, t)$ is the Green function of Eq. (6) (i.e., the solution for a Dirac delta boundary condition centered at $x=0$ and
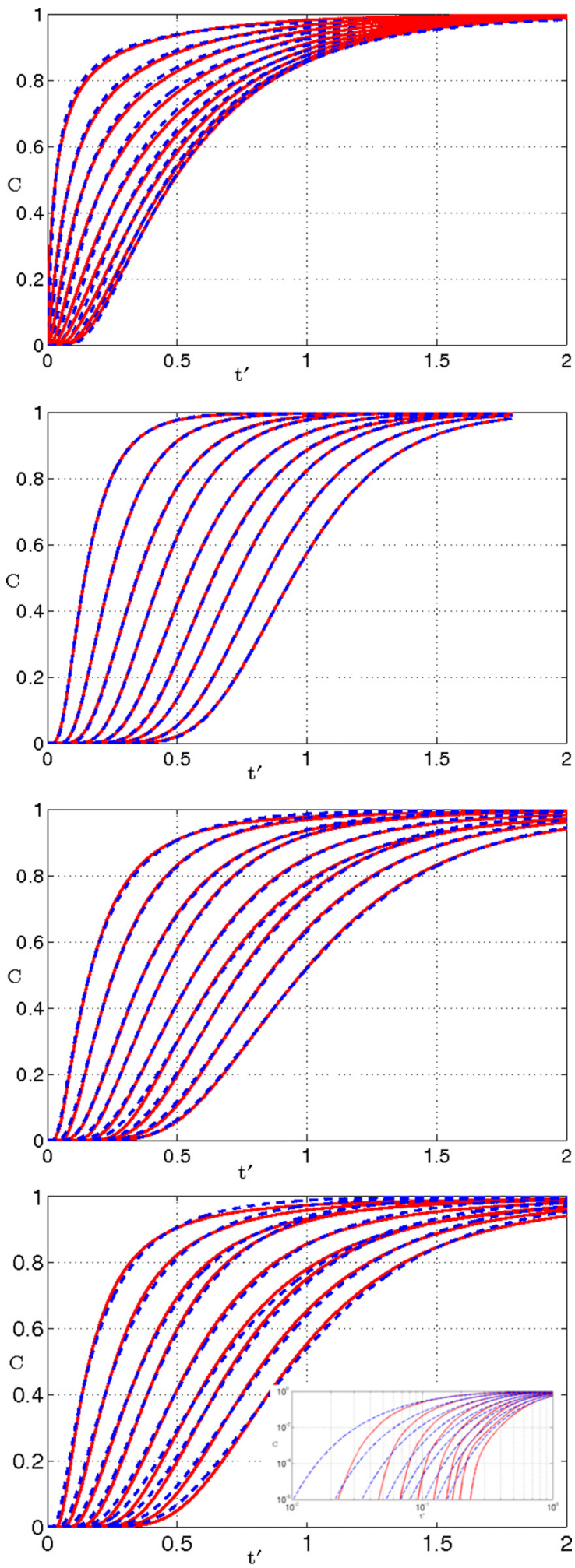

FIG. 11. (Color online) Comparison between breakthrough curves (normalized dimensionless solute concentration, continuous red line) over dimensionless time $t^{\prime}=t \epsilon / V_{x}$ obtained by surface averaging over equidistant sections of the porous medium for different Péclet numbers (from top to bottom, $\mathrm{Pe}=1.9 \times 10^{-1}$, $\left.\mathrm{Pe}=1.9 \times 10^{1}, \mathrm{Pe}=1.9 \times 10^{3}, \mathrm{Pe}=1.3 \times 10^{5}\right)$ with the analytic solutions of the semi-infinite advection-diffusion equation with the fitted parameters (dashed blue line). For the largest Pe number an insert in log-log scale is included. 


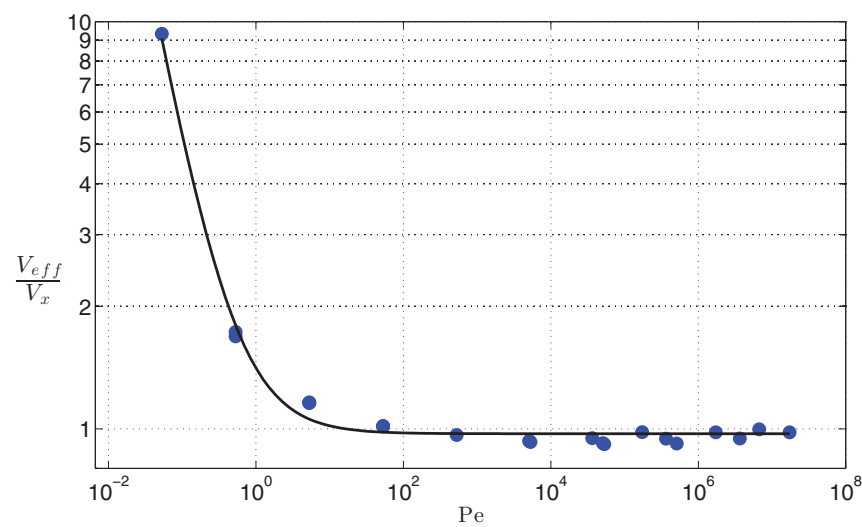

FIG. 12. (Color online) Effective transport velocity, as estimated by the method of moments, and its fitting, of the form $a+b / \mathrm{Pe}$, with $a=0.97$ and $b=0.15$.

$t=0)$. The effective velocity is then given by

$$
V_{\mathrm{eff}}=\left(\frac{\epsilon \mathcal{L}_{x}}{M_{1}}\right),
$$

whereas the dispersion coefficient is given by

$$
D=\frac{M_{2}}{2 \mathcal{L}_{x}}\left(\frac{V_{x}}{\epsilon}\right)^{3} .
$$

This method in general performs better for the estimations of porosity (or equivalently the Darcy fluxes) and hydrodynamic dispersion in terms of computational time, accuracy and stability. Since the two methods resulted, however, in very similar (if not almost identical) results, only the parameters estimated with the method of moments will be shown and discussed.

It is moreover interesting to observe that the simulated breakthrough curves, reported in Fig. 11, compare well with the analytical solutions with the parameters estimated with the method of moments. However, some small differences are observed, especially in the case of large Péclet numbers. To highlight these differences, of primary interest when it comes to anomalous diffusion, an insert in log-log scale has been added.

Figure 12 shows the estimation of the effective velocity ( $V_{\text {eff }}$ ) divided by the value of $V_{x}$ computed from the averaging for the different Péclet numbers. As can be seen, the effect of diffusive flux [see Eq. (13)] is important for low Péclet numbers when they can be orders of magnitude larger than the advective fluxes. When this effect is no more visible (i.e., for high Péclet numbers), the estimated velocity is always slightly lower than the theoretical one. This is clearly explained by Eq. (13) where the gradient of porosity appears as an additional convective flux (in our case in fact the porosity in the last sections used for the fitting is about $3 \%$ lower than the initial one).

Figure 13 shows the estimated dispersion coefficients $D$ divided by the molecular diffusion coefficient $D_{m}$ for the different Péclet numbers investigated. It should be observed that the scaling of $D / D_{m}$ with the Péclet number reproduces many recent computational results and classical power laws (obtained in turn by experimental and theoretical studies [29,31,59]). As is seen a constant behavior is observed in the first region (very low Pe), followed by a superlinear

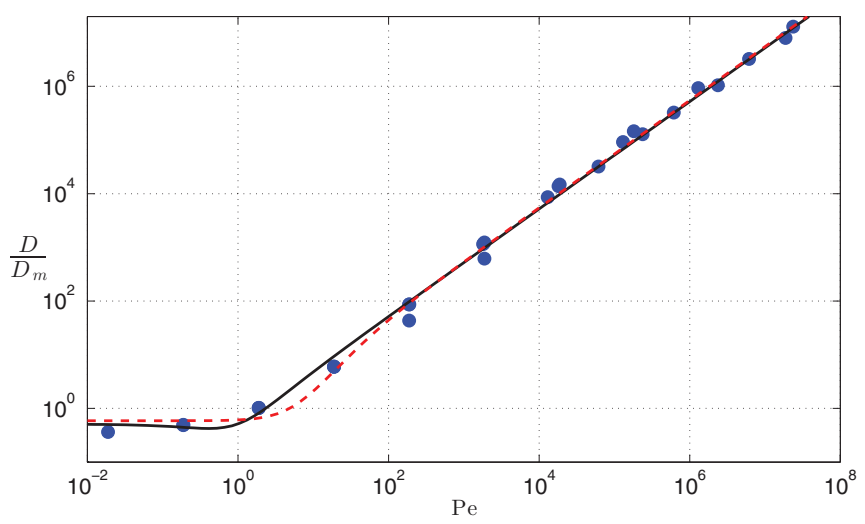

FIG. 13. (Color online) Dispersion coefficients for different Péclet numbers, as estimated by the method of moments (blue circles) together with two fitted correlation laws; the continuous black line is Eq. (17), whereas the dashed red line is Eq. (18).

relationship (with an exponent of 1.2) in the central part, and a linear scaling at very high Pe in the last part.

It is interesting to notice that in the constant part $(\mathrm{Pe} \rightarrow 0)$ of the plot, the result of Eq. (14), is confirmed. In fact, for this system the tortuosity was quantified to be about 1.2 , resulting in a ratio between $D$ and $D_{m}$ of about 0.69 (as observable in Fig. 13). Considering the linear regime (high Péclet numbers) the already introduced expression: $D=\alpha V_{x}$, was found to be an adequate description for our medium; the constant $\alpha$ was estimated to be between $60 \%$ and $90 \%$ of $d_{50}$, depending on the Péclet number.

Eventually, if the data of $D / D_{m}$ versus Pe are fitted with the laws reported in Eqs. (17) and (18), the following results are obtained. The fitted parameters for Eq. (17) are $\gamma=0.51$, $\beta=0.52$, and $\mathrm{Pe}_{c}=0.99$, whereas for Eq. (18) are $\gamma=0.59$, $\alpha=1.52 \times 10^{-4}$, and $\delta=1.76$. It is interesting to highlight that the coefficient $\gamma$ estimated with these two correlations are in good agreement with each other and with the estimation of tortuosity given by Eqs. (14) and (15). Both laws are reported (with these values of $\gamma, \alpha, \beta, \delta$, and $\mathrm{Pe}_{c}$ ) in Fig. 13 together with our simulation results.

Last, it is interesting to verify the validity of Fickian diffusion, which was used to close Eq. (12). In order to perform this analysis the quantity $\left\langle v_{x}^{\prime} c^{\prime}\right\rangle$, reported on the left-hand side of Eq. (12), was calculated and compared with the quantity reported on the right-hand side of Eq. (12) for 10 different sections. This latter quantity is the product of the dispersion coefficient and the gradient on the main flow direction $(x)$ of the surface-averaged concentration, $C(x)$, or in other words the dispersive flux as approximated by Fick's law, with the value of $D_{\mathrm{L}}$ calculated with the fitting performed with the method of moments. Typical results of this analysis for two superficial velocities are reported together with the dispersive fluxes at four different instants of the simulations in Fig. 14, and, as is possible to see, the two curves are very close at low superficial velocity $\left(\operatorname{Re}=9.6 \times 10^{-3}\right)$. Larger deviations from the theoretical Fickian behavior are noticeable for higher velocities $(\operatorname{Re}=66)$, where vorticity and wakes develop more extensively. However, for both cases the hypothesis of Fickian behavior can be safely assumed. 

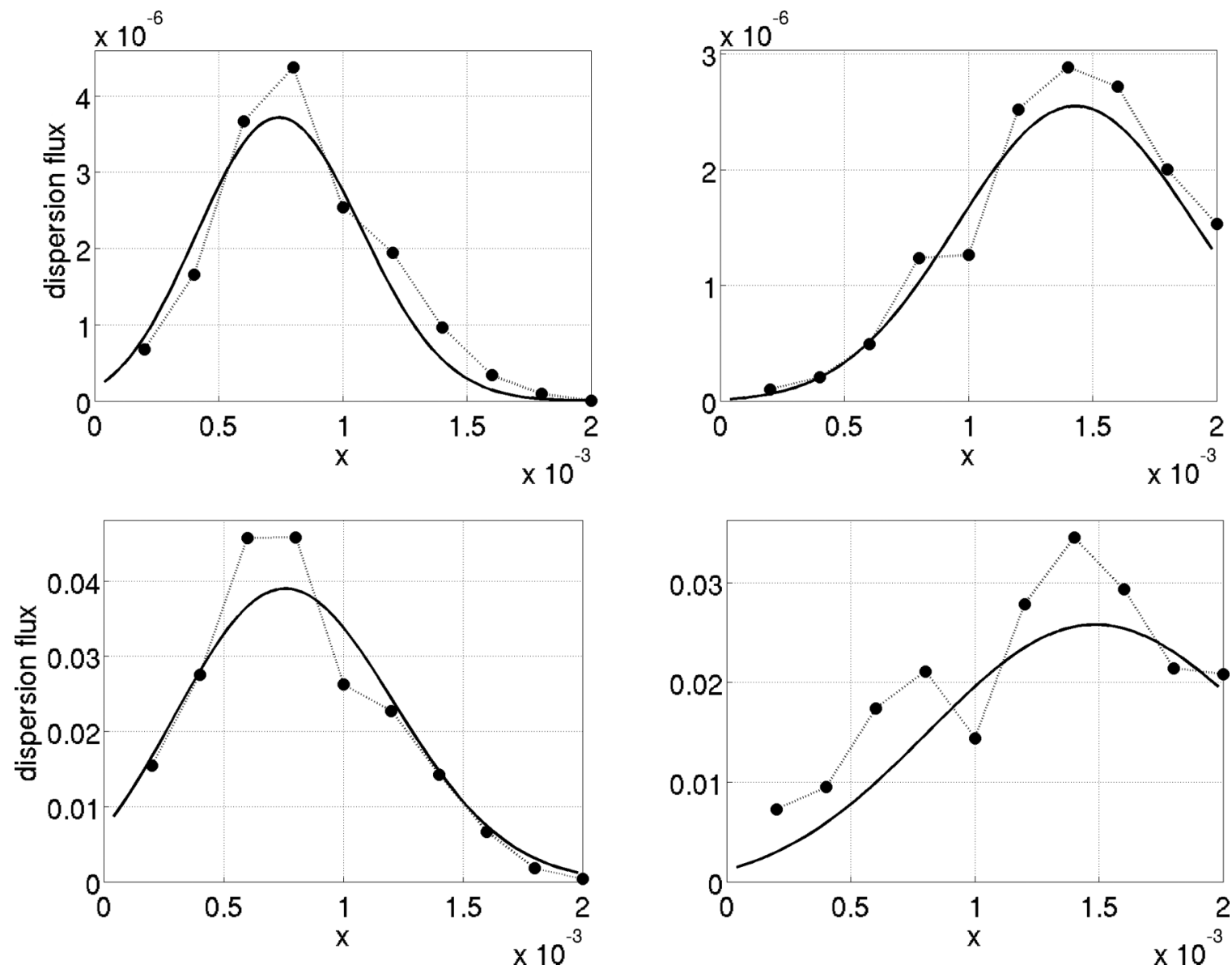

FIG. 14. Comparison of the dispersion flux $\left\langle v_{x}^{\prime} c^{\prime}\right\rangle\left(\mathrm{m} \mathrm{s}^{-1}\right)$ along the flow $x$ coordinate $(\mathrm{m})$ as calculated from the three-dimensional simulations (dotted lines with symbols) and as approximated by Fick's law (computed as the spatial derivative of the analytic solution for semi-infinite media with the fitted parameters, continuous line) for two different Reynolds numbers (top: $\operatorname{Re}=9.6 \times 10^{-3}$; bottom: $\operatorname{Re}=66$ ) and at two instants (from left to right).

\section{SUMMARY AND CONCLUSIONS}

Pore-scale simulations of single phase flows and scalar transport have been carried out by means of CFD with highorder numerical schemes and advanced meshing techniques. Wide ranges of Reynolds (from $10^{-4}$ to $10^{2}$ ) and scalar Péclet (from $10^{-2}$ to $10^{6}$ ) numbers have been investigated, including nonlinear regimes. Results from steady-state (for flow field) and transient (for transport) simulations have been extracted on a hundred mesh slices perpendicular to the $x$ axis. Permeability, mean tortuosity, and mean shear rate have been calculated explicitly from the flow field results, while the dispersivity has been estimated with post-processing tools based on the method of moments and on the least square formulation of the inverse problem.

The results demonstrate the validity of the method, predicting the linear and nonlinear regimes of Darcy's law with welldefined permeability and tortuosity constants in the first regime and three different regimes for hydrodynamic dispersion [60]: the first one is dominated by the molecular dispersion (for low velocity and fine particles), then a region where the mechanical and molecular dispersion are of the same order of magnitude, and finally a region where the dispersion depends linearly on the Péclet number, where the inertial effects dominate. This correlation for the hydrodynamic dispersion in terms of Péclet number is verified for the porous medium under study with the proposed simulation and upscaling tools.

The asymptotic dispersion regime is quickly reached, and dispersion is well approximated by the Fickian hypothesis, even if the fluid velocity distributions are not Gaussian. Further studies will include a more careful characterization of the influence of the porous structures in terms of porosity and tortuosity, and numerical upscaling of more complex macroscopic models will also be considered.

\section{ACKNOWLEDGMENTS}

Funding for this work has been partially provided by the project PRIN 2008 "Produzione, stabilizzazione e trasporto di nano-particelle di ferro zero-valente per bonifica di acquiferi contaminati" of the Italian Ministero dell' Istruzione, Università e Ricerca. Computational facilities have been partially offered by a European PRACE project, by the Texas Supercomputing Center, by the KAUST Visualization Lab, and by the KAUST HPC Lab. The authors acknowledge Dmitri Naumov for providing the code "SettleDyn", Dario Forneris for suggesting the use of the code BLENDER, and Eleonora Crevacore for her work regarding the grid-independence analysis. 
[1] A. M. Tartakovsky, Phys. Rev. E 82, 026302 (2010).

[2] S. Whitaker, The Method of Volume Averaging (Kluwer Academic Publishers, Dordrecht, 1999).

[3] B. Berkowitz, J. Bear, and C. Braester, Water Resour. Res. 24, 1225 (1995).

[4] J. Nordbotten, M. Celia, H. Dahle, and S. Hassanizadeh, Water Resour. Res. 43, W08430 (2007).

[5] P. Forchheimer, Z. Ver. Deut. Ing. 45, 1782 (1901).

[6] T. Tosco, D. L. Marchisio, F. Lince, and R. Sethi, Transport Porous Med. 96, 1 (2013).

[7] R. Sethi, J. Hydrol. 400, 187 (2011).

[8] A. D. Molfetta and R. Sethi, Ingegneria degli acquiferi (Springer, Milan, 2012).

[9] M. Rolle, G. Chiogna, D. L. Hochstetler, and P. K. Kitanidis, J. Contam. Hydrol. 153, 51 (2013).

[10] R. D. Bauer, M. Rolle, S. Bauer, C. Eberhardt, P. Grathwohl, O. Kolditz, R. U. Meckenstock, and C. Griebler, J. Contam. Hydrol. 105, 56 (2009).

[11] J. D. Hyman, P. K. Smolarkiewicz, and C. L. Winter, Phys. Rev. E 86, 056701 (2012).

[12] Y. Wang, Phys. Rev. E 87, 032144 (2013).

[13] B. Bijeljic, A. Raeini, P. Mostaghimi, and M. J. Blunt, Phys. Rev. E 87, 013011 (2013).

[14] P. Parthasarathy, P. Habisreuther, and N. Zarzalis, Chem. Eng. Sci. 90, 242 (2013).

[15] B. Bijeljic, A. H. Muggeridge, and M. J. Blunt, Water Resour. Res. 40, W1150101 (2004).

[16] G. Garmeh, R. Johns, and L. Lake, SPE J. 14, 559 (2009).

[17] S. Khirevich, A. Höltzel, and U. Tallarek, Comm. Comput. Phys. 13, 801 (2013).

[18] R. S. Maier, D. M. Kroll, R. S. Bernard, S. E. Howington, J. F. Peters, and H. T. Davis, Phys. Fluids 12, 2065 (2000).

[19] P. Mostaghimi, B. Bijeljic, and M. Blunt, SPE J. 17, 1131 (2012).

[20] S. Ovaysi and M. Piri, J. Contam. Hydrol. 124, 68 (2011).

[21] Y. Zhu and P. J. Fox, J. Comput. Phys. 182, 622 (2002).

[22] F. Augier, F. Idoux, and J. Delenne, Chem. Eng. Sci. 65, 1055 (2010).

[23] M. J. Blunt, B. Bijeljic, H. Dong, O. Gharbi, S. Iglauer, P. Mostaghimi, A. Paluszny, and C. Pentland, Adv. Water Resour. 51, 197 (2013).

[24] G. Boccardo, D. Marchisio, and R. Sethi, J. Colloid Interf. Sci. 417, 227 (2014).

[25] J. Bear, Dynamics of Fluids in Porous Media (Dover, New York, 1988).

[26] M. Balhoff, A. Mikelić, and M. F. Wheeler, Transport Porous Med. 81, 35 (2010).

[27] K. Rajagopal, Math. Models Methods Appl. Sci. 17, 215 (2007).

[28] S. Whitaker, AIChE J. 13, 420 (1967).

[29] J. Bear and Y. Bachman, in IASH Symposium in Artificial Recharge and Management of Aquifers (IASH Publ., Haifa, 1967), Vol. 72, pp. 7-16.
[30] U. Hornung, Homogenization and Porous Media (Springer, New York, 1997).

[31] M. Sahimi, Flow and Transport in Porous Media and Fractured Rock (Wiley, Weinheim, 2012).

[32] D. A. Benson, S. W. Wheatcraft, and M. M. Meerschaert, Water Resour. Res. 36, 1403 (2000).

[33] J. H. Cushman and T. R. Ginn, Water Resour. Res. 36, 3763 (2000).

[34] B. Berkowitz, A. Cortis, M. Dentz, and H. Scher, Rev. Geophys, 44, RG2003 (2006).

[35] A. M. Tartakovsky, D. M. Tartakovsky, and P. Meakin, Phys. Rev. Lett. 101, 044502 (2008).

[36] R. Haggerty and S. M. Gorelick, Water Resour. Res. 31, 2383 (1995).

[37] H. Gerke and M. v. Genuchten, Water Resour. Res. 29, 305 (1993).

[38] A. Koponen, M. Kataja, and J. Timonen, Phys. Rev. E 54, 406 (1996).

[39] M. B. Clennell, Geol. Soc., Lond., Spec. Pub. 122, 299 (1997).

[40] A. Duda, Z. Koza, and M. Matyka, Phys. Rev. E 84, 036319 (2011).

[41] B. P. van Milligen and P. D. Bons, Phys. Rev. E 85, 011306 (2012).

[42] J. Feder, J. Theor. Biol. 87, 237 (1980).

[43] M. Pilotti, Transport Porous Med. 33, 257 (1998).

[44] C. L. Y. Yeong and S. Torquato, Phys. Rev. E 57, 495 (1998).

[45] P.-E. Øren and S. Bakke, Transport Porous Med. 46, 311 (2002).

[46] P.-S. Koutsourelakis and G. Deodatis, J. Eng. Mech. 131, 397 (2005).

[47] R. J. Adler and J. E. Taylor, Random Fields and Geometry (Springer, New York, 2007).

[48] E. Coumans, bulletphysics.org (2006).

[49] G. Blöcher and G. Zimmermann, Comput. Geosci. 34, 1827 (2008).

[50] J. Van Gumster, Blender for Dummies (Wiley, Indianapolis, 2009).

[51] W. E. Lorensen and H. E. Cline, in ACM Siggraph Computer Graphics (ACM, New York, 1987), Vol. 21, pp. 163-169.

[52] P. Cignoni, M. Callieri, M. Corsini, M. Dellepiane, F. Ganovelli, and G. Ranzuglia, in Eurographics Italian Chapter Conference (Eurographics Association, Salerno, 2008), p. 129.

[53] OpenCFD, The Open Source CFD Toolbox, User Guide (OpenCFD (ESI), London, 2013).

[54] J. H. Cushman, L. S. Bennethum, and B. X. Hu, Adv. Water Resour. 25, 1043 (2002).

[55] R. Hill and D. Koch, J. Fluid Mech. 465, 59 (2002).

[56] R. Hill, D. Koch, and A. Ladd, J. Fluid Mech. 448, 243 (2001).

[57] R. Hill, D. Koch, and A. Ladd, J. Fluid Mech. 448, 213 (2001).

[58] R. Aris, Proc. Roy. Soc. Lond. A 235, 67 (1956).

[59] J.-L. Auriault and P. Adler, Adv. Water Resour. 18, 217 (1995).

[60] M. Sahimi, AIChE J. 41, 229 (1995). 